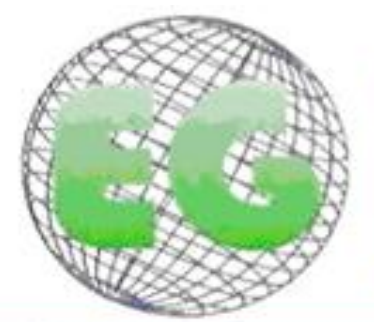

\title{
Conductas problema en adolescentes en la ciudad de Monterrey, México
}

Problem behaviors in adolescents in the city of Monterrey, Mexico

\section{*Díaz Rodríguez, Cecilia Lourdes **González Ramírez, Mónica Teresa}

*Facultad de Psicología. Universidad Autónoma de Nuevo León. Monterrey, NL México. E-mail: cecydiaz_@hotmail.com

Palabras clave: Adolescencia; conductas problema; teoría de la conducta problema.

Keywords: Adolescence, problem behaviors, problem behaviors theory.

\section{RESUMEN}

En este trabajo se comparan las encuestas de población objetivo de información aplicadas en México en relación a las conductas problema de los adolescentes (alcohol, tabaco, drogas, sexo desprotegido, accidentes y hábitos alimenticios), comparándolas con el Cuestionario de Conductas Problema mismo que mide la presencia o ausencia de las conductas problema, así como la aparición de dichas conductas en los ambientes en los que se desenvuelve el adolescente (familia, amigos, vecindario y escuela). Se utilizó un diseño no experimental, transversal, descriptivo y se evalúan las conductas problema de 551 adolescentes de primer semestre $(M=14.8$ años, $D T=2.35)$ de nivel medio superior de una institución pública de la Ciudad de Monterrey, N.L. México. Los resultados muestran que es necesario analizar los fenómenos desde una encuesta descriptiva más amplia que involucre los ambientes en los que se lleva a cabo el fenómeno medido con el fin de entender las necesidades de cada población, así como realizar programas psicoeducativos dirigidos a las necesidades detectadas.

\begin{abstract}
In this paper, we compare the surveys that we applied to the Mexico population in relation to problem behaviors in adolescents (alcohol, snuff or tobacco, drugs, unprotected sex, accidents and eating habits), as compared to the Problem Behaviors Questionnaire which measures the presence or absence of problem behaviors and the occurrence of these behaviors in the environments in which it operates teenager (family, friends, neighborhood and school). We used a non-experimental, crosssectional, descriptive and evaluated the problem behaviors of 551 adolescents in first semester $(\mathrm{M}=$ 14.8 years, $S D=2.35$ ) high school level of a public institution of the City of Monterrey, NL Mexico. The results show that it is necessary to analyze the phenomena from a larger descriptive survey involving the environments in which they carried out the phenomenon measured in order to understand the needs of each population as well as perform psychoeducational programs aimed at the needs identified.
\end{abstract}




\section{INTRODUCCIÓN}

La adolescencia es una etapa caracterizada por los cambios importantes que se producen en ella, cambios físicos, cognitivos y sociales ${ }^{(1-6)}$. El desarrollo de estos cambios está ligado al proceso de adaptación y búsqueda de identidad; y es en este proceso que hacen su aparición los factores de riesgo y/o protección, tema central del presente artículo.

Conger y Peterson ${ }^{(7)}$ señalan que la adolescencia comienza como biología y termina como cultura, por lo que conciben este periodo como un proceso de cambios biológicos, psicológicos y sociales que se relacionan entre sí y que permiten a los individuos alcanzar su identidad personal y su integración a la sociedad.

La adolescencia se caracteriza por ser clave en la adquisición de los estilos de vida, saludables o problemáticos. La adquisición de un mayor nivel de independencia del ambiente familiar y un mayor compromiso con el grupo de iguales puede llevar a los adolescentes a nuevas situaciones donde se enfrentan a la toma de decisiones que pueden afectar su futuro; en esta etapa los jóvenes pueden incurrir en conductas problema como fumar, beber, uso de drogas, iniciar su vida sexual, tener relaciones sexuales desprotegidas o bajo el influjo de alcohol o drogas, manejar a exceso de velocidad o bajo el influjo del alcohol.

Sin embargo es importante definir y explicar la diferencia que existe entre factores de riesgo y conductas de riesgo, ambos conceptos tomados en cuenta para la realización de esta investigación. Pineda y Aliño ${ }^{(8)}$ definen los factores de riesgo como los efectos con altas probabilidades de daño o resultados no deseados para el adolescente, sobre los cuales se debe de actuar. Alba ${ }^{(9)}$ define conducta de riesgo como cualquier comportamiento que comprometa los aspectos biopsicosociales del desarrollo exitoso del adolescente. Dicho de otra manera, los factores de riesgo son el escenario donde se desarrollan las conductas problema o conductas de riesgo (el término conducta de riesgo será sustituida por conducta problema en la presente investigación).

La Teoría de la Conducta Problema (TCP) enfatiza como comportamientos problemáticos el uso de alcohol, problemas de alcoholismo, tabaquismo, consumo de marihuana, otras drogas ilícitas, conducta desviada en general (conductas delictivas y la norma de otros actos violatorios), la conducción de riesgo, y las relaciones sexuales precoces $^{(10-15)}$.

\section{Conductas Problema}

En México la Encuesta Nacional de Salud y Nutrición (ENSANUT) ${ }^{(16)}$ ofrece información que constituye un diagnóstico actualizado de las condiciones de salud de los mexicanos, así como sobre la respuesta del sistema de salud a estas condiciones, y los resultados alcanzados. Realiza una medición descriptiva de las conductas problema de los adolescentes y presenta los siguientes datos:

En relación a la conducta de tabaquismo definida como la conducta realizada por aquel individuo que había fumado 100 cigarros o más en su vida, se encontró que el $9.2 \%$ de los adolescentes consumen tabaco, además se reportó que la edad de inicio del 50\% fue entre los 13 y 15 años. La Encuesta Nacional de Adicciones (2011) (17) reporta una prevalencia de fumadores activos de $12.3 \%$, la encuesta también reporta 
que los fumadores adolescentes activos que fuman diariamente inician su consumo en promedio a los 14.1 años, siendo similar para hombres y mujeres. La edad de inicio ha permanecido estable de 2002 a 2011.

El consumo de alcohol se define como el consumo de una bebida alcohólica de forma diaria, semanal, mensual u ocasional en el último año; los datos de la ENSANUT (2012) muestran que el $25 \%$ de los adolescentes (hombres y mujeres) consumen alcohol. La ENA (2011) encontró que el consumo de alcohol aumentó significativamente en las tres prevalencias medidas, de tal manera que el consumo "alguna vez" pasó de $35.6 \%$ a $42.9 \%$, "en el último año" de $25.7 \%$ a $30.0 \%$ y "en el último mes" de $7.1 \%$ a $14.5 \%$. Esta misma tendencia se observó en los hombres y en las mujeres, especialmente en el consumo del último mes ya que se incrementó en el caso de ellos de $11.5 \%$ a $17.4 \%$ y en ellas de $2.7 \%$ a $11.6 \%$

La ENA (2011) muestra que las prevalencias de consumo de drogas en zona urbana más altas siguen siendo la mariguana (1.6\%), la cocaína $(0.5 \%)$ y los inhalables $(0.4 \%)$. Estos datos muestran un ligero aumento en comparación con la misma encuesta aplicada en el 2008, sin embargo el incremento no es significativo. Específicamente en la región norte del país, existe una prevalencia de $2.2 \%$ del consumo de marihuana

En relación a la sexualidad, en 2012 el 90\% de la población de 12 a 19 años de edad reporta conocer o haber escuchado hablar de algún método anticonceptivo, cifra que aumentó de $82 \%$ y $69 \%$ en 2006 y 2000 respectivamente. El debut sexual reporta un aumento tanto en hombres como en mujeres de 12 a 19 años; el 25.5\% de los hombres y el $20.5 \%$ de las mujeres ha iniciado su vida sexual. Del total de adolescentes sexualmente activos, $14.7 \%$ de los hombres y $33.4 \%$ de las mujeres no utilizaron ningún método anticonceptivo en la primera relación sexual. En cuanto al embarazo adolescente, los resultados muestran que del total de las mujeres adolescentes de 12 a 19 años de edad que tuvieron relaciones sexuales, la mitad (51.9\%) alguna vez ha estado embarazada.

Los accidentes en adolescentes (sin ser exclusivos los accidentes al manejar) se encuentra que la cifra disminuyó de $8.8 \%$ a $8.3 \%$ entre la aplicación de las encuestas y se encuentra que el grupo con mayor riesgo de sufrir accidentes es el de 10 a 12 años tanto en hombres como en mujeres. La prevalencia de intento de suicidio en adolescentes se incrementó con respecto a la registrada en la ENSANUT 2006, de $1.1 \%$ a $2.7 \%$. Lo mismo ocurre en las prevalencias según sexo, al pasar de 0.6 a $0.9 \%$ en el grupo de hombres y de 1.7 a $4.6 \%$ en las mujeres.

Las conductas alimentarias de riesgo más frecuentes en adolescentes mexicanos fueron: preocupación por engordar, comer demasiado y perder el control sobre lo que se come. En las adolescentes, la principal conducta de riesgo fue la preocupación por engordar (19.7\%). En los varones, la conducta de riesgo más frecuente fue hacer ejercicio para perder peso (12.7\%).

Sin embargo, las encuestas aplicadas a la población mexicana como la ENA, ENSANUT, o encuesta especial aplicada por el Instituto Nacional de Estadística, Geografía e Informática (INEGI) arrojan datos puramente descriptivos que solamente permiten observar el fenómeno y el incremento o decremento que ha tenido cierta conducta, y no nos permite analizar los cambios mismos en las conductas vinculados 
a los cambios de la sociedad, cambios en su estructura, valores, creencias y ambientes relacionados de manera directa con los cambios del fenómeno.

Buscando encontrar más información para entender el funcionamiento de las conductas problema se realizó una evaluación en adolescentes de preparatoria utilizando un instrumento diseñado por Jessor ${ }^{(18)}$ desde la TCP, misma que el elaboró. El Cuestionario de Conductas Problema (CCP) tiene un formato descriptivo al igual que las encuestas aplicadas actualmente, sin embargo aporta la ventaja de analizar los ambientes (familia, amigos, escuela y vecindario) que pueden ser escenario de las conductas de los adolescentes (alcoholismo, tabaquismo, drogadicción, sexo desprotegido, accidentes y hábitos alimenticos). El objetivo principal del estudio es mostrar los datos obtenidos con el nuevo instrumento así como realizar un contraste de resultados en el estado de Nuevo León comparándolos con los datos obtenidos por la ENSANUT 2012 y ENA 2011 a nivel nacional.

\section{METODOLOGÍA}

\section{Participantes}

Participaron en el estudio 551 adolescentes de entre 14 y 19 años, siendo en su mayoría de 15 años (79\%), 50\% son hombres. En relación a la familia se encontró que el $77 \%$ vive con ambos padres, sin embargo, el $11 \%$ reporta que sus padres están separados y $8 \%$ menciona tener padres divorciados; $18 \%$ de ellos viven en un hogar monoparental.

\section{Diseño}

Se realizó un estudio no experimental de corte transversal-descriptivo, teniendo como objetivos elaborar una medición de las conductas problema de los adolescentes de manera real y acertada, midiendo la presencia de la conducta además de la función de los ambientes (riesgo o protección) llamándole un diagnóstico específico para una población específica.

\section{Instrumento}

Se utilizó el instrumento elaborado por Richard Jessor (2001) Adolescent Health and Development Questionnaire, que consta de 276 ítems. Evalúa las conductas problema propuestas en la TCP (alcoholismo, tabaquismo, drogadicción, sexo desprotegido, conducta delictiva, manejo de riesgo y hábitos alimenticios) cada una de estas conductas problema es medida de manera exploratoria-descriptiva y en cada uno de los ambientes del adolescente (familia, amigos, escuela, vecindario) para determinar si estos ambientes son de riesgo o protección para cada una de las conductas problema.

El lenguaje original del instrumento es el inglés y se realizó una traducción al español en Bolivia, sin embargo la traducción no se adaptaba a la ciudad de Monterrey y fue traducido para nuestra sociedad siguiendo el procedimiento de traducción a la inversa. 


\section{Análisis estadístico}

Se utilizó el paquete estadístico IBM SPSS versión 21 para el análisis de los datos y se presentan datos descriptivos.

\section{Aspectos éticos}

Se solicitó autorización al director de la Escuela. Considerando que los participantes eran menores de edad se les solicitó que firmaran un consentimiento y se permitió retirarse a quienes no quisieran participar en la investigación. Asimismo se les aseguró que sus respuestas serían manejadas con total discreción y sus datos personales de identificación serían confidenciales.

\section{RESULTADOS}

Se describen a continuación los resultados obtenidos con CCP por cada una de las conductas problema y se realiza la comparación con los datos de las encuestas nacionales:

\section{Tabaco}

Del total de 551 participantes, 96 han probado el cigarro alguna vez en su vida y 79 fumaron en los últimos 12 meses anteriores a la aplicación de la encuesta. Se le preguntó a este grupo de fumadores acerca del consumo regular ( $\begin{array}{ll}1 & 0\end{array}$ veces por semana) del tabaco, $40 \%$ mencionó que no fuma regularmente, $20 \%$ muy pocas veces, $23 \%$ algunas veces y $17 \%$ la mayoría del tiempo.

Se les cuestionó por la cantidad de cigarro que fuman en un día, se encontró que el $37 \%$ no fuma diariamente, $35 \%$ fuma menos de un cigarro, $20 \%$ entre 1 y 3 cigarros, $4 \%$ entre 4 y 8 cigarros, $3 \%$ una cajetilla al día y $1 \% 3$ cajetillas o más (ver tabla I). La edad de debut fue de 14 años (36\%), seguida de $13(22 \%)$ y 12 años (17\%).

Tabla I: Cantidad de cigarros fumados diariamente

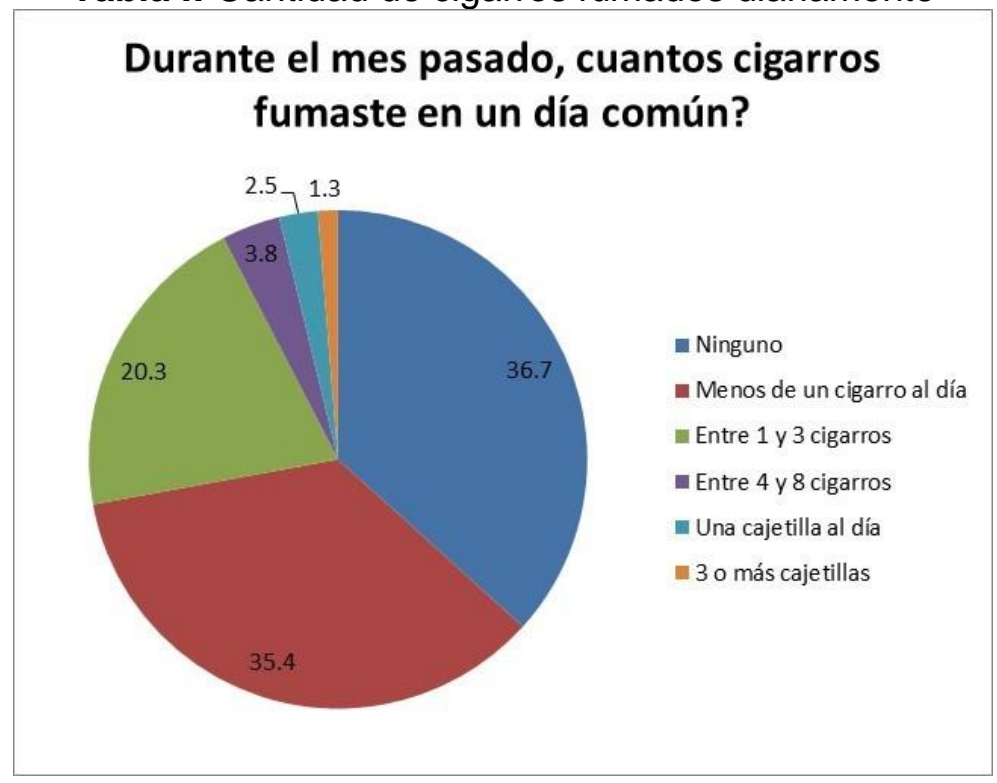

Se analizaron también los ambientes del adolescente en busca de la percepción de dichos ambientes como un riesgo o una protección. 
En su grupo de amigos se encontró que según el $79 \%$ sus pares no los presionan para fumar, seguido del $23 \%$ que opinan que los presionan un poco, $5 \%$ una buena cantidad y $1 \%$ siente que presionan mucho. Se les cuestionó la aceptación del hábito de fumar entre su grupo de amigos; $54 \%$ lo aprueban y $30 \%$ lo desaprueban (ver tabla II):

Tabla II: Opinión de pares acerca de fumar

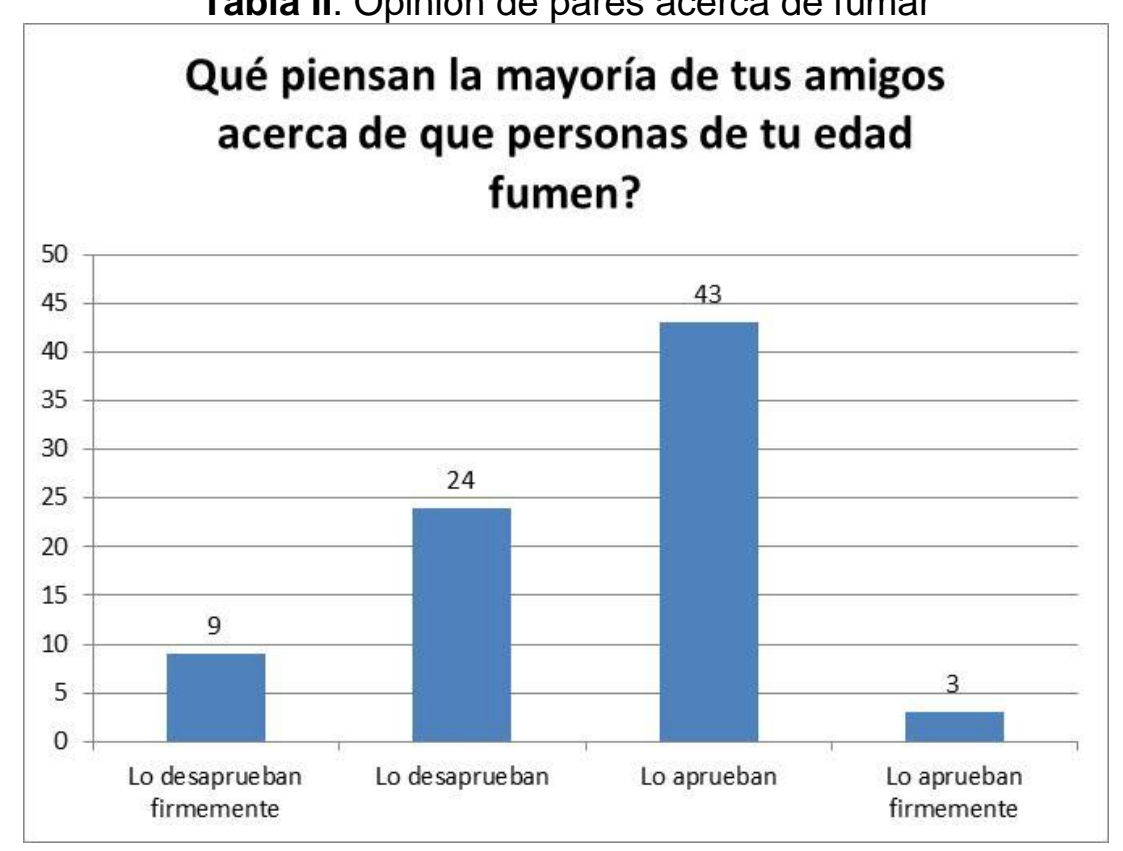

Así mismo se cuestionó acerca del consumo del grupo de amigos, el 6\% de los adolescentes no tiene amigos que fumen, $64 \%$ algunos amigos, $24 \%$ la mayoría de sus amigos y $5 \%$ casi todos sus amigos fuman.

La familia de la mayoría de los adolescentes reprueba la conducta de fumar; se cuestionó si sus padres supieran que han estado fumando tendrían problemas, el $57 \%$ dice que definitivamente, $33 \%$ piensa que probablemente, $9 \%$ probablemente no y solo el $1 \%$ definitivamente no. Sin embargo, el $38 \%$ de los papas así como el $30 \%$ de las mamás de los adolescentes fuma. Se les preguntó si podrían conseguir cigarros en casa, $68 \%$ definitivamente no conseguiría, $13 \%$ probablemente no, $17 \%$ probablemente sí y $3 \%$ definitivamente.

Dentro del vecindario el adolescente percibe una buena cantidad de consumo de tabaco $(37 \%)$, un poco consumo (37\%), mucho consumo (19\%) y nada de consumo $(6 \%)$.

Por último se les pregunto a los jóvenes si piensan que fumar puede tener efectos en la salud, los resultados se presentan en la siguiente tabla (ver tabla III): 
Tabla III: Opinión de efectos de la conducta de fumar

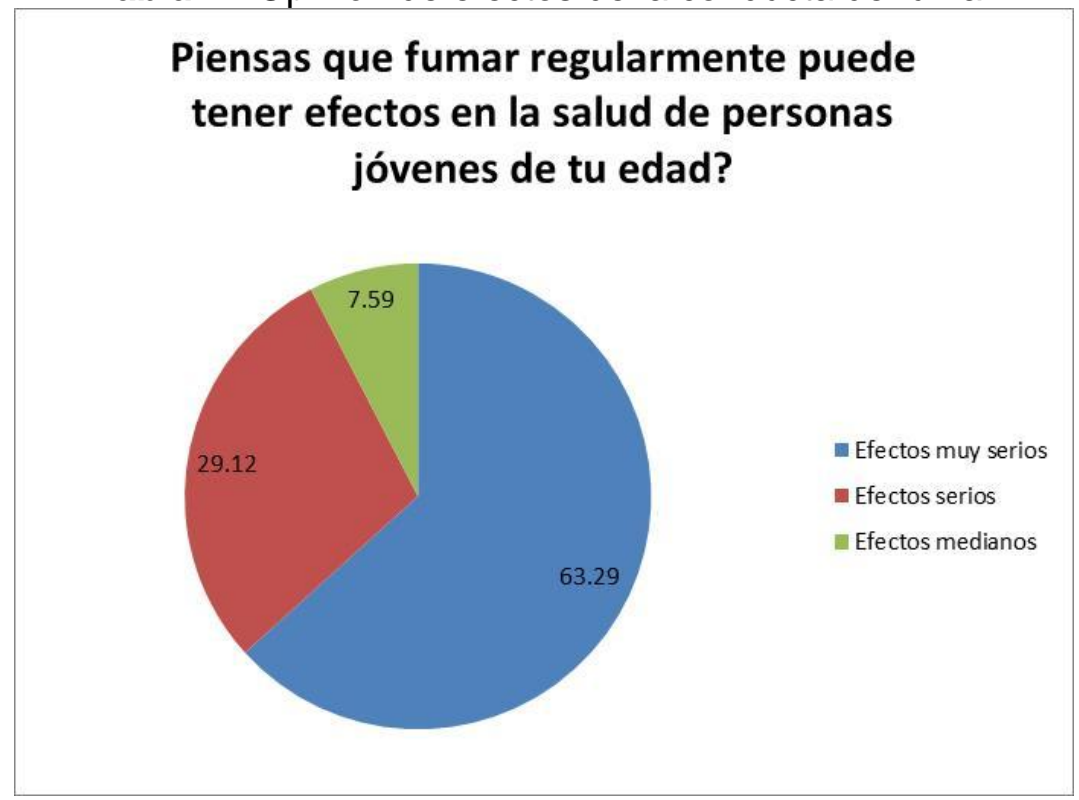

Comparando los datos obtenidos con el CCP y la ENSANUT (2012), se encontró con el CCP que el $14.3 \%$ de los adolescentes consumen tabaco, contra el $9.2 \%$ de los adolescentes a nivel nacional.

\section{Alcohol}

208 de los 551 participantes han consumido alcohol de manera regular en los últimos 6 meses. 52\% no contesto su edad de debut, sin embargo el 15.4\% bebió por primera vez a los 14 años, seguido de un $12 \%$ a los 15 años.

En relación a la frecuencia en la conducta de beber, se encontró que el $31 \%$ de los participantes en los últimos 6 meses había bebido 3 o 4 veces, seguido de un $26 \%$ que no había bebido nada. Sin embargo el $27.5 \%$ ha bebido en un rango frecuente que va desde 1 vez al mes hasta todos los días (ver tabla IV).

Tabla IV: Consumo de alcohol en los últimos 6 meses

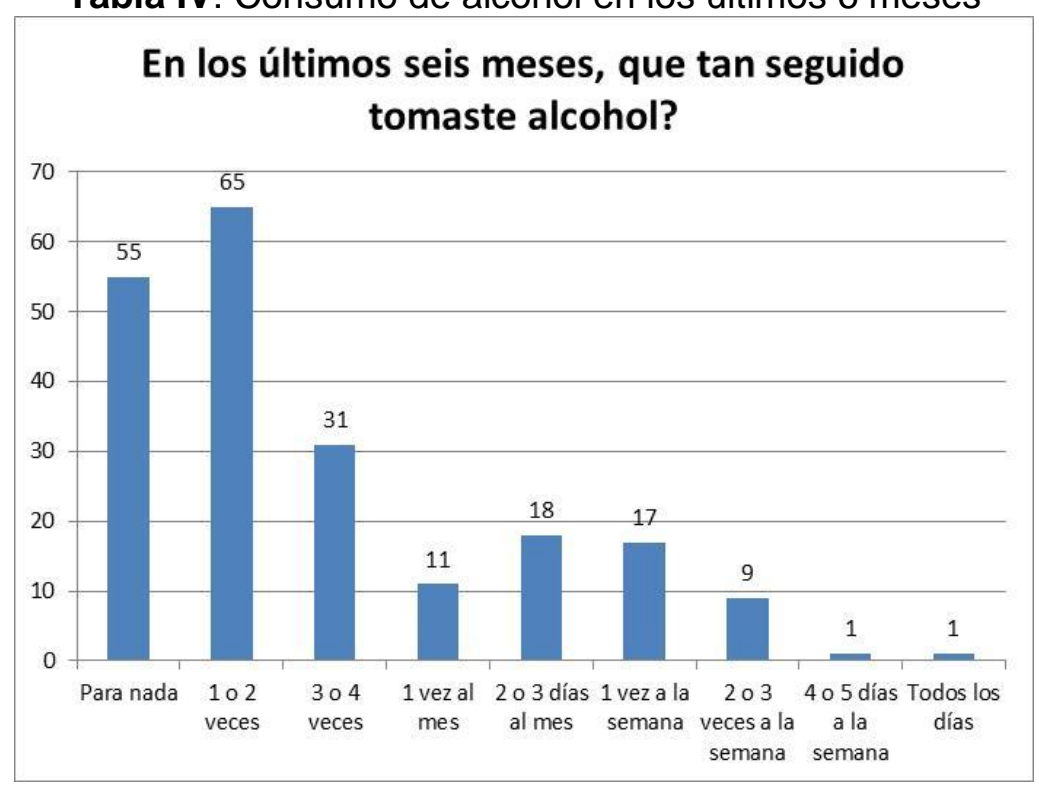


El consumo de bebidas por ocasión en que los adolescentes beben muestra que $27 \%$ no contestó la pregunta, el $38.5 \%$ consume entre menos de una bebida y dos bebidas, sin embargo el $34.6 \%$ de los adolescentes consumen entre tres y nueve bebidas o más (ver tabla $\mathrm{V}$ ).

Tabla V: Cantidad de alcohol consumida en los últimos seis meses

Qué cantidad has tomado en cada ocasión que has tomado en los últimos 6 meses?

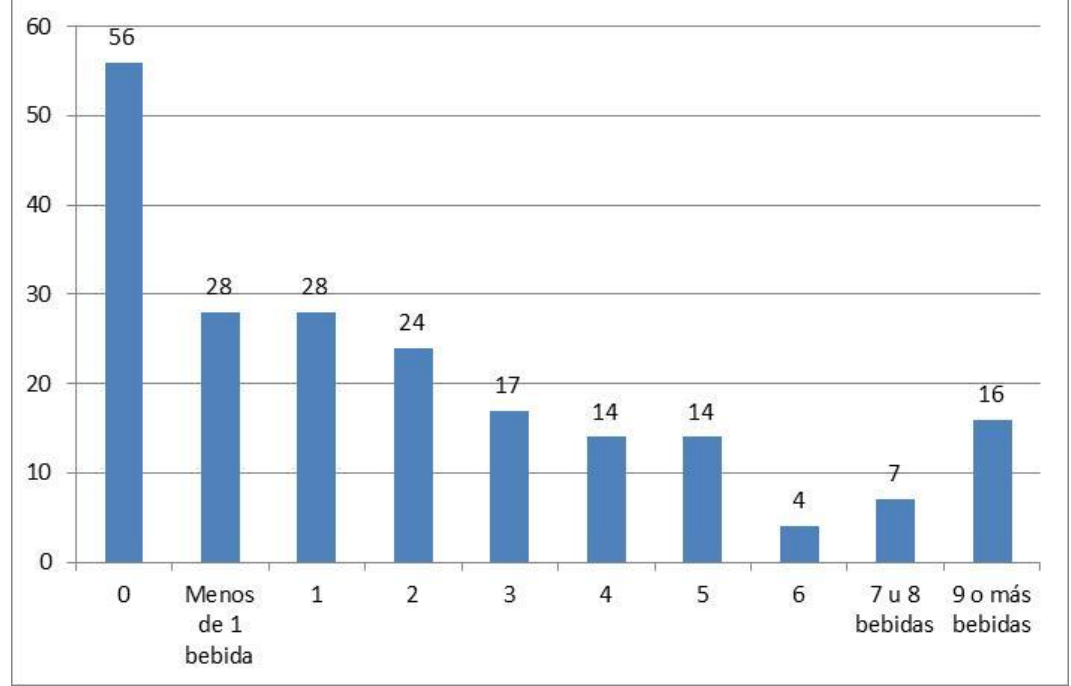

Se preguntó al adolescente si a causa de beber alcohol habían tenido problemas con sus padres, escuela, amigos, alguien con quien salían o policía, se reportan los datos de aquellos que han tenido problemas al menos una vez hasta 5 o más veces (ver tabla VI):

Tabla VI: Problemas ocasionados por beber alcohol con:

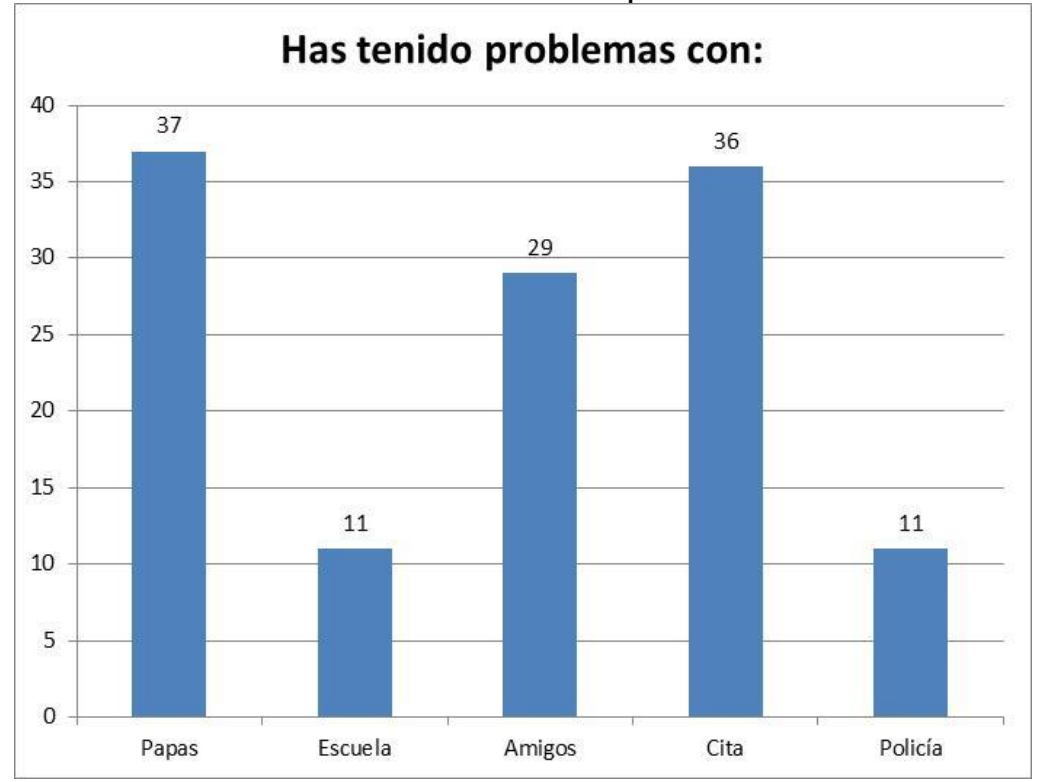

Al preguntar al adolescente cuántas veces se había emborrachado, se encontró que el $26 \%$ no contestó, $42 \%$ nunca y $31 \%$ se ha emborrachado al menos una vez hasta más de dos veces a la semana. 
Se analizaron también los ambientes del adolescente en busca de la percepción de dichos ambientes como un riesgo o una protección.

En relación a los amigos y la presión social que se ejerce para la ingesta de alcohol, el $62 \%$ de los adolescentes opinan que sus amigos no ejercen nada de presión para que beban alcohol, $32 \%$ opina que presionan un poco y $6 \%$ opinan que presionan una buena cantidad o mucho. Al preguntarles acerca de qué opina su grupo de amigos de la conducta de beber, la respuesta estuvo polarizada (52.5\% lo aprueban contra $47.2 \%$ lo desaprueban) y específicamente de su entorno de amistades se les preguntó cuántos de sus amigos toman alcohol de manera regular, se encontró que solamente $10 \%$ reporta que ninguno lo hace, $63 \%$ menciona que algunos lo hacen, $20 \%$ la mayoría de ellos y $6.7 \%$ casi todos ellos.

Acerca del ambiente familiar, se preguntó a los adolescentes qué pasaría si sus padres supieran que han estado tomando sin permiso, se encontró que más del $50 \%$ de ellos piensa que definitivamente tendría problemas (ver tabla VII). Y en relación a la accesibilidad en casa, se encontró que $51 \%$ opina que definitivamente no podría conseguir alcohol en casa, $26 \%$ probablemente no podría conseguirlo, $18 \%$ probablemente podría y solo $3.8 \%$ definitivamente podría conseguirlo.

Tabla VII: Aceptación de padres respecto al consumo de alcohol

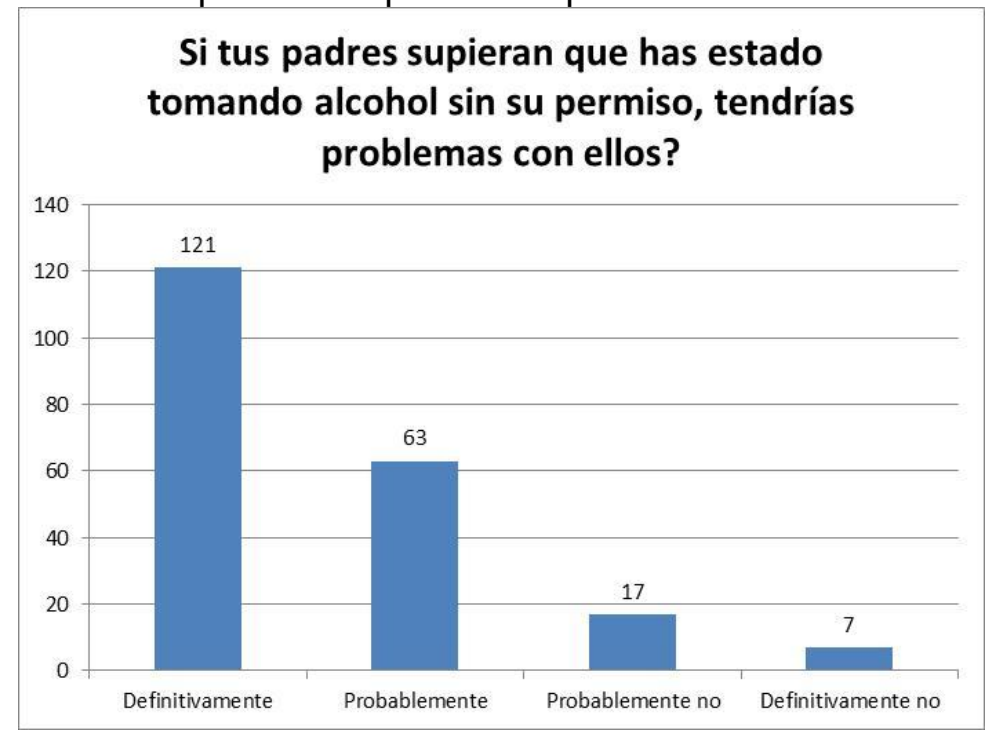

Y por último el ambiente del vecindario, al preguntar al adolescente cuánto consumo de alcohol existe entre los adultos en la colonia donde vive, el $60 \%$ opina que mucho o una buena cantidad. Y en relación a la accesibilidad en el vecindario, se encontró en este ambiente una respuesta polarizada, $51.5 \%$ piensa que definitivamente 0 probablemente podría conseguir alcohol en el vecindario y $49.5 \%$ piensa que definitivamente y probablemente no.

Se les realizó a los adolescentes una última pregunta de opinión acerca de los efectos a la salud que puede tener el consumo de alcohol de manera regular, se encontró lo siguiente (ver tabla VIII): 
Tabla VIII: Opinión de efectos de la conducta de beber

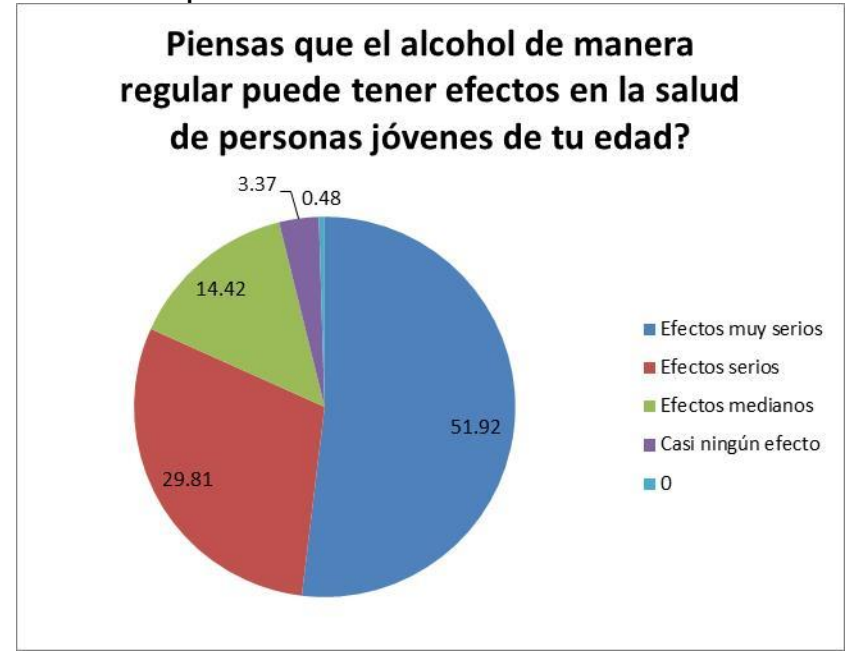

En relación al consumo de alcohol el $25 \%$ a nivel nacional de hombres y mujeres consumen alcohol, en Monterrey el $38 \%$ de los adolescentes consumen y $28 \%$ de ellos han consumido desde 3 veces hasta todos los días en los últimos 6 meses.

\section{Drogas}

Para conocer el acercamiento a las drogas se les preguntó a los adolescentes si habían probado la mariguana, ya que es la principal droga de inicio. 95\% nunca la ha probado, $4 \%$ una vez y $1 \%$ más de una vez. La edad de inicio principal fue a los 15 años, sin embargo existe un caso de debut a los 11 años (ver tabla IX).

Tabla IX: Edad de debut de mariguana

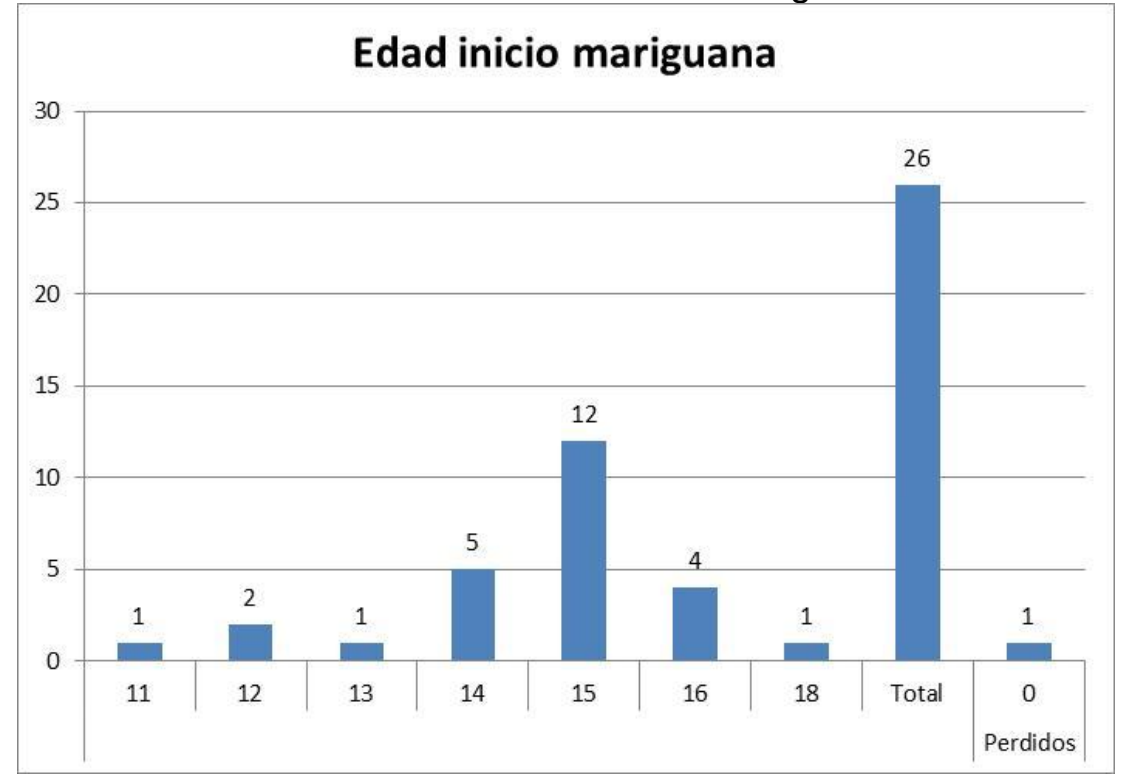

Se les cuestionó la frecuencia del consumo de mariguana, 44\% no la ha usado en 6 meses, $30 \%$ una vez, $15 \% 2-3$ veces y $12 \%$ entre 4 veces y todos los días.

A los usuarios de mariguana se les cuestionó en relación a sus amistades, en primer lugar por la opinión de su conducta; $70 \%$ lo desaprueba, $19 \%$ lo aprueba y $11 \%$ lo desaprueba firmemente. $Y$ en relación al consumo de sus amigos se encontró que 
$67 \%$ menciona que algunos de sus amigos también consumen, seguido del $19 \%$ que ninguno de ellos lo hacen, $11 \%$ muchos de ellos y $4 \%$ casi todos ellos consumen.

Al cuestionarles acerca de su familia, preguntando qué pasaría si sus padres supieran que han estado usando mariguana o alguna otra droga el $85 \%$ piensa que definitivamente tendría problemas, $11 \%$ probablemente tendría problemas y $4 \%$ definitivamente no tendría problemas.

El consumo de drogas en el vecindario es percibido por el adolescente usuario como: nada $(56 \%)$, un poco $(26 \%)$, mucho $(11 \%)$ y una buena cantidad $(7 \%)$. Y el acceso en el vecindario es de $26 \%$ por igual para: definitivamente no, probablemente no y probablemente si y $22 \%$ definitivamente si podría conseguir.

Por último se le preguntó los efectos en la salud del consumo de drogas, los resultados se presentan en el siguiente gráfico (tabla X):

Tabla X: Opinión de efectos del consumo de mariguana

Piensas que el uso continuo de mariguana puede tener serios efectos en la salud de personas jóvenes de tu edad?

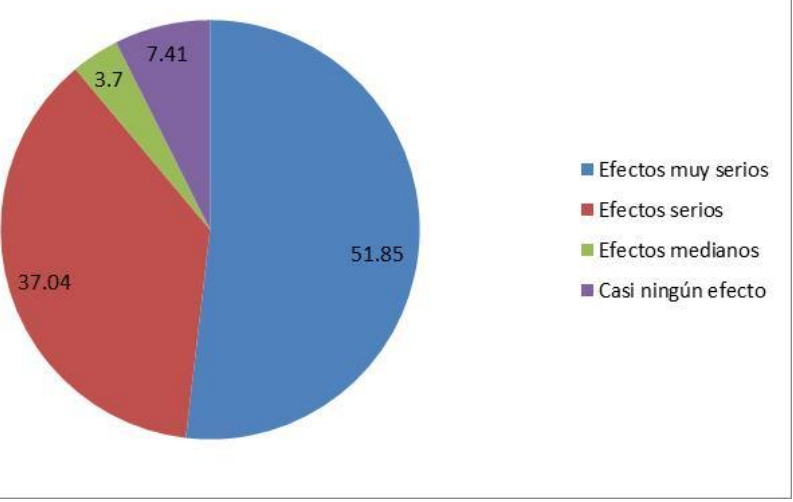

Se observa un incremento en los datos obtenidos con el CCP es el consumo de mariguana a nivel estatal, ya que un $5 \%$ de los adolescentes dicen haberla probado, contra un $1.6 \%$ a nivel nacional.

\section{Sexualidad}

Se preguntó a los adolescentes acerca de su vida sexual. Para comenzar se les preguntó cómo describirían su identidad sexual, $83 \%$ reporta ser heterosexual, $1 \%$ es bisexual y $9 \%$ no está seguro, nadie contestó homosexual. El $80 \%$ no ha tenido relaciones sexuales, $14 \%$ si ha tenido y $6 \%$ no contestó (ver tabla XI). 
Tabla XI: Debut sexual

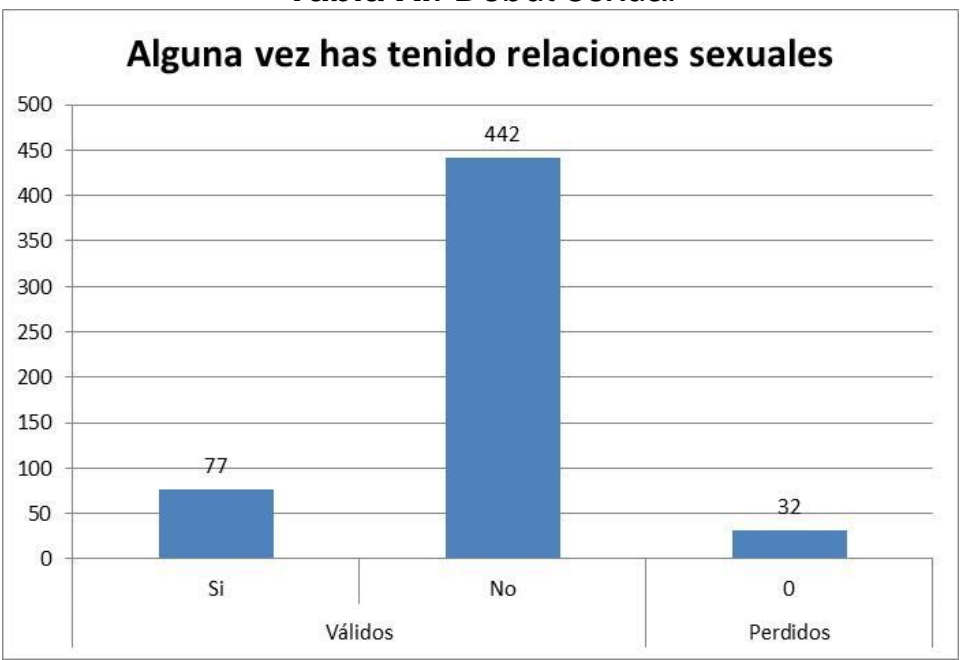

Al cuestionarles acerca de la edad de inicio sexual se encontraron como resultados principales que el $48 \%$ inició su vida sexual a los 15 años, $32 \%$ a los 14 años y $9 \%$ a los 13 años. Se les preguntó también si esa primera vez que tuvieron relaciones sexuales sintieron que estaban obligados, los resultados muestran que el $94 \%$ no se sintió obligado, $5 \%$ si se sintió obligado y $1 \%$ no contestó. Así mismo se les preguntó si se habían embarazado o habían embarazado a alguna chica alguna vez, 95\% respondió que no, 1\% mencionó que una vez y $1 \%$ más de una vez.

La presión entre los pares para tener relaciones sexuales es percibida por los adolescentes como "nada" en un 66\%, "un poco" fue mencionado por el $21 \%, 6 \%$ considera que existe una buena cantidad de presión y por último existe un $2 \%$ que reporta mucha presión para iniciar la vida sexual. Sin embargo, se les preguntó a los adolescentes cuántos de sus amigos han tenido relaciones sexuales, $53 \%$ reportan que solamente algunos de sus amigos han tenido relaciones sexuales, seguido del $33 \%$ que menciona que ninguno de sus amigos han tenido relaciones sexuales; los menores porcentajes se encuentran en un $7 \%$ que menciona que la mayoría ellos y solamente un $2 \%$ casi todos ellos.

Las conductas sexuales muestran datos inferiores a los presentados en las encuesta aplicadas a nivel nacional: en relación al inicio sexual se encuentra con el CCP que el $22 \%$ de los hombres y $6 \%$ de las mujeres han iniciado su vida sexual, la ENSANUT (2012) encuentra que el $25.5 \%$ de los hombres y $20.5 \%$ de las mujeres han iniciado su vida sexual. También se encontró que la edad de inicio sexual para la muestra de Monterrey es de un año menos que el promedio nacional (12-15 vs 13-15 años)

Un dato más que se encuentra en las encuestas nacionales es que el $51.9 \%$ de las mujeres adolescentes que tuvieron relaciones sexuales han estado embarazadas, en la ciudad se encuentra que solamente el .04\% de las chicas ha estado embarazada 0 los chicos han embarazado a alguna chica.

\section{Accidentes}

Los accidentes en esta encuesta están relacionados con las conductas al conducir. 224 adolescentes han manejado un carro. Se les cuestionó acerca de las horas en las que conducen, $67 \%$ menciona que no conduce nada de noche, $22 \%$ conduce algo, $6 \%$ casi la mitad del tiempo y $4 \%$ la mayoría del tiempo (ver tabla XII). 
Tabla XII: Horas de conducción en horario nocturno

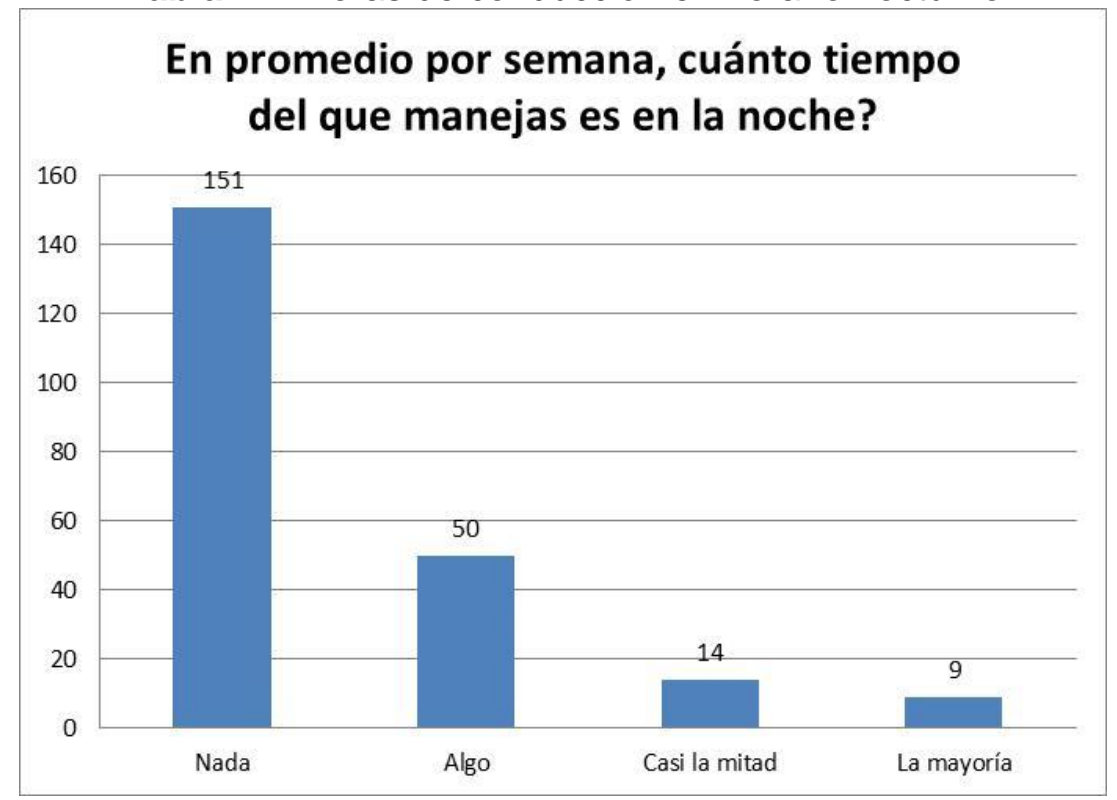

Se preguntaron conductas específicas de riesgo que pueden desencadenar en un accidente, por ejemplo, si manejan por una señal de alto sin detenerse completamente, $89 \%$ mencionó que nunca lo hace, $8 \%$ lo ha hecho una o dos veces, $2 \% 6$ o más veces y $0.5 \%$ de 3 a 5 veces. Al preguntar acerca de si mantienen una distancia prudente entre su carro y el carro de enfrente se encontró que $84 \%$ menciona que nunca maneja muy cerca, $12 \%$ una o dos veces lo ha hecho, $2 \%$ de 3 a 5 veces y $2 \% 6$ o más veces. Se les preguntó también si habían manejado después de haber tomado al menos una cerveza u otra bebida alcohólica, 93\% nunca lo ha hecho, $5 \%$ una o dos veces, $0.5 \%$ de 3 a 5 veces y $1 \% 6$ o más veces. Una conducta más de riesgo es el manejar a una velocidad superior a $80 \mathrm{~km} / \mathrm{hra}, 69 \%$ nunca lo ha hecho, $21 \%$ una o dos veces, $4 \%$ de 3 a 5 veces, y $7 \% 6$ veces o más, y la última conducta de riesgo al manejar es pasarse un semáforo en rojo, $89 \%$ nunca lo ha hecho, $6 \%$ lo ha hecho una o dos veces, $2 \%$ de 3 a 5 veces, y $2 \%$ lo ha hecho 60 más veces.

En esta conducta problema no se cuestionaron los ambientes del adolescente para detectar riesgo o protección, ya que se consideran como factores personales.

La última conducta de riesgo analizada son los hábitos alimenticios, el $32 \%$ de los adolescentes encuestados se salta el desayuno la mayoría de las mañanas, 34\% algunas mañanas y $30 \%$ casi nunca. Se les preguntó qué tan frecuentemente consumían un snack en lugar de su comida regular, casi el 50\% lo hace algunas veces, $30 \%$ casi nunca y $15 \%$ la mayor parte del tiempo.

En relación a los hábitos alimenticios de la familia, se encontró que el $64 \%$ opina que algunos miembros de su familia consumen comida chatarra, $23 \%$ menciona que ninguno de sus familiares consume comida chatarra, $6 \%$ piensa que la mayoría de ellos y $2 \%$ que casi todos ellos lo hacen.

Se les preguntó también acerca del consumo de comida chatarra por parte del grupo de amigos, $51 \%$ menciona que algunos de ellos lo hacen, $32 \%$ que la mayoría lo hace, $8 \%$ casi todos ellos la consumen, y $4 \%$ ninguno de ellos. 
Y por último se les preguntó su opinión acerca de los efectos a futuro de una mala conducta alimenticia, específicamente al saltarse el desayuno, el 35\% piensa que puede tener efectos serios, $29 \%$ efectos medianos, $23 \%$ efectos muy serios y $8 \%$ casi ningún efecto. $Y$ de los efectos de consumir comida chatarra, el $44 \%$ piensa que puede tener efectos muy serios, $37 \%$ efectos serios, $12 \%$ efectos medianos y sólo $3 \%$ casi ningún efecto.

Las encuestas nacionales y el CCP realizan preguntas diferentes en relación a los hábitos alimenticios y no hay preguntas de contaste.

\section{DISCUSIÓN}

Cada una de las conductas problema presenta factores de riesgo y protección similares, por ejemplo lo biológico, social y ambiental. Las principales son el consumo de los padres, la edad, la aceptación de los padres, abuso sexual durante la infancia o la niñez, el grado de estudios, exposición a la sustancia, disfuncionalidad familiar y comorbilidad con el consumo de otras sustancias ${ }^{(19-23)}$.

La teoría de la conducta problema ${ }^{(18)}$ considera como factores de riesgo y protección lo biológico/genético, el medio social, el medio percibido, la personalidad y la conducta. El CCP permite evaluar el medio percibido y la conducta. El análisis de ambientes de los adolescentes encuestados nos permite establecer un diagnóstico de las conductas problema y los ambientes en los que se desenvuelve.

En la conducta problema del consumo de alcohol se encontró que los amigos no se perciben como un ambiente de riesgo, ya que no se percibe presión de pares, sin embargo se percibe consumo entre el grupo de amigos y eso puede ser un factor desencadenante para el consumo. La familia es un factor de protección, el adolescente reporta que si la familia sabe que bebe alcohol tendría problema y también reporta que no tiene acceso a alcohol en su casa. Y por último el vecindario, se diagnosticó como un ambiente de riesgo por la cantidad de consumo percibido, así mismo el $50 \%$ de los adolescentes reportó que podría conseguir alcohol en su colonia.

La conducta problema de fumar se comporta de manera similar a la conducta problema de consumir alcohol. Se encuentra que el adolescente no percibe presión del grupo de pares, sin embargo muestran aceptación del consumo y muchos de los amigos fuman. La familia es un ambiente de protección, el adolescente percibe que tendría problemas si su familia sabe que fuma y un buen porcentaje de papás y mamás fuman. $\mathrm{Y}$ el vecindario se diagnostica como un riesgo, ya que se percibe mucho consumo en él.

El consumo de drogas (específicamente la mariguana) es la conducta problema donde se encontró que los amigos son un ambiente de riesgo, ya que un porcentaje alto aprueban la conducta y casi el $82 \%$ de ellos tiene amigos consumidores. La familia sigue siendo un ambiente percibido como protección ya que reporta desaprobar la conducta y el vecindario es detectado como un ambiente de riesgo donde hay accesibilidad y un consumo percibido.

En relación a la sexualidad, se analizó solamente el ambiente social, y se encontró que los amigos son un ambiente de riesgo, existe una buena cantidad de presión para iniciar su vida sexual, sin embargo el grupo de pares no ha iniciado su vida sexual. 
Los accidentes, los cuales fueron analizados a través de la conducta al manejar y no a través de ningún ambiente, se encontró que la conducta de riesgo que los adolescentes presentan con mayor frecuencia es el manejar a más de $80 \mathrm{kms} / \mathrm{hra}$. Y pocos, aunque suficientes, han bebido y manejado, esto puede relacionarse con el ambiente social.

Y por último los hábitos alimenticios, donde se encontró que la familia es un factor de protección, ya que se percibe como poco consumidora de comida chatarra, a diferencia del grupo social, el cual es percibido como consumidor de comida chatarra.

Los adolescentes con mayor vulnerabilidad a incurrir en conductas de alto riesgo tienen problemas en múltiples ámbitos, y tienden a pertenecer a redes sociales que potencian y refuerzan el desarrollo de estos modelos de conducta. Por lo que, cuanto mayor sea el número de factores de riesgo a los que se expone un adolescente, mayor será la probabilidad de que se convierta en un menor infractor

\section{CONCLUSIONES}

Se encuentran datos descriptivos en un nivel que permite la creación de programas psicoeducativos dirigidos al adolescente mismo en relación a las conductas problema que se detectaron con mayor riesgo, así mismo se permite la creación de nuevos modelos de intervención en los ambientes de riesgo y en las conductas de riesgo específicas.

Se recomienda ampliar las preguntas de algunas conductas problema como la sexualidad y los accidentes, así como incluir preguntas que permitan la correlación y predicción de factores de riesgo y conductas problema.

\section{REFERENCIAS}

1 Barra, E., Cerna, R., Kramm, D. y Váliz, V. (2006). Problemas de salud, estrés, afrontamiento, depresión y apoyo social en adolescentes. Terapia Psicolóogica, 24, (1), 55-61.

2 Aliño, M., López, J. y Navarro, R. Adolescencia, aspectos generales y atención a la salud. Revista Cubana Med Gen Integr 2006; 22, (1).

3 Ulanowicz, M., Parra, K., Wendler, G y Monzón, L. Riesgos en el embarazo adolescente. Revista de Posgrado de la VI Cátedra de Medicina, 153.

4 González-Garza, C., Rojas-Martínez, R., Hernández-Serrato, M y Olaiz Fernández, G. Perfil del comportamiento sexual en adolescentes mexicanos

de 12 a 19 años de edad. Resultados de la ENSA 2000. Salud Pública de México 2005; 47(3).

5 Casco, F.J. y Oliva, A. Valores y expectativas sobre la adolescencia: discrepancias entre padres, profesores, mayores y adolescentes. Infancia y aprendizaje 2005; 28(2), 209-220.

6 Motrico, E., Fuentes, M y Bersabé, R. Discrepancias en la percepción de los conflictos entre padres e hijos/as a lo largo de la adolescencia. Anales de Psicología 2001; 17(1), 1-13.

7 Aragón, L.E. \& Bosques, E. Adaptación familiar, escolar y personal del adolescente de la ciudad de México. Enseñanza e Investigación en Psicología 2012; 17(2), 263-282.

8 Pineda, S. \& Aliño, M. Manual de prácticas clínicas para la atención integral 
a la salud en la adolescencia. Cuba: MINSAP; 2002.

9 Alba, L. Salud de la adolescencia en Colombia: Bases para una medicina de prevención. Universidad Médica de Bogotá 2010; 51(1), 29-42.

10 Donovan, J.E., Jessor, R. y Costa, F.M. Adolescent Health Behavior and Conventionality-Unconventionality: An extension of Problem-Behavior Theory. Health Psychology 1991; 10(1), 52-61.

11 Jessor, R., Van Den Bos, J., Costa, F.M. y Turbin, M.S. Protective Factors in Adolescent Problem Behavior: Moderator Effects and Developmental Change Developmental Psychology 1995; 31(6), 923-933.

12 Jessor, R., Turbin, M.S. y Costa, F.M. Predicting Developmental Change in Risky Driving: The Transition to Young Adulthood. Applied Developmental Science 1997; 1(1), 4-16.

13 Jessor, R., Turbin, M.S. y Costa, F.M. Risk and Protection in Successful Outcomes Among Disadvantaged Adolescents. Applied Developmental Science 1998a; 2(4), 194-208.

14 Jessor, R., Turbin, M.S. y Costa, F.M. Protective Factors in Adolescent Health

Behavior. Journal of Personality and Social Psychology 1998b; 75(3), 788 800.

15 Pastor, Belaguer y García-Merita Dimensiones del estilo de vida relacionado con la salud en la adolescencia: Una revisión. Revista de Psicología General

y Aplicada 1998; 51(3,4), 469-483.

16 Instituto Nacional de Salud Pública. Encuesta Nacional de Salud y Nutrición 2012. [Internet], Mexico: Secretaria de Salud Publica; 2012; [Acceso 13 Junio

2013].

http://ensanut.insp.mx/informes/ENSANUT2012ResultadosNacionales.pdf

17 Instituto Nacional de Psiquiatría Ramón de la Fuente Muñiz/Instituto Nacional

de Salud Pública 2011. Encuesta Nacional de Adicciones 2011: Reporte de Drogas. [Internet], Mexico: [Acceso 13 en Junio de 2013.]

http://www.conadic.salud.gob.mx/pdfs/ENA 2011 DROGAS ILICITAS .pdf

18 Jessor, R. Risk Behavior in Adolescence: A Psycosocial Framework for Understanding and Action. Journal of Adolescent Health 1991; 12, 597-605.

19 Kendler, K., Sundquist, K.,Ohlsson, H., Palme'r, K., Maes, H., Winkleby, M.A., et al. Genetic and Familial Environmental Influences on the Risk for Drug Abuse. ARCH GEN PSYCHIATRY 2011; 69(7), 690-697.

20 Kendler, K., Gardner, Ch. \& Prescott, C. Toward a Comprehensive Developmental Model for Alcohol Use Disorders in Men. Twin Res Hum Genet 2011; 14(1), 1-15.

21 Acosta, L., Fernández, A. \& Pílón, S. Factores sociales para el uso de alcohol en adolescentes y jóvenes. Rev. Latino-Am Enfermagem 2011; 19, 771-781.

22 Alfonso, J.P., Huedo-Medina, T.B. \& Espada, J.P. Factores de riesgo predictores del patrón de consumo de drogas durante la adolescencia. Anales de Psicología 2009; 25(2), 330-338.

23 Navarro, E., Vargas, R., Martínez, R., Padilla, B., Ruiz, D. \& Thorne, B. Factores asociados al consumo de cigarrillo en adultos del suroccidente de Barranquilla, Colombia. Salud Uninorte. Barranquilla (Colombia) 2005; 21,314. 
ISSN 1695-6141

(C) COPYRIGHT Servicio de Publicaciones - Universidad de Murcia 